\title{
Wave functions in the laboratory
}

\section{A clever measurement of the radiative decay of excited molecular hydrogen has made possible direct estimates of a vibrational wave function, perhaps pointing the way to new tests of old calculations.}

PEOPLE calculate wave functions all the time, but can they measure them? The diffraction pattern of an electron beam scattered by a metal may be a good representation of the wave function of a free electron, but that is hardly startling. Now an ingenious piece of spectroscopy of the humble hydrogen molecule by W. Koot, W. J. van der Zande and J. Los (Phys. Rev. Lett. 58, 2746; 1987), from the Dutch Institute for Atomic and Molecular Physics at Amsterdam, seems to have broken new ground in the visualization of a bound-state wave function.

The measurement is that of the spontaneous disintegration of a highly excited electron state of molecular hydrogen which, being a triplet (parallel electron spins), is inaccessible from the singlet ground state, but whose decay leads predominantly to the separation of the two atoms and the emission of a photon. If that were all there is to say, the measurement would be simply that of the frequency of the photon. But molecules have vibrationally excited states as well so that, in a bound-free transition, the vibrational energy is partitioned between the energy of the photon and the kinetic energy of the separating atoms.

Much of the cleverness of the experiment lies in the preparation of hydrogen molecules in a pure state in which the vibrational as well as the electron quantum numbers are known. In the end, Los and his colleagues are able to use a measurement of the energy carried away by the separating hydrogen atoms to calculate the shape of the vibrational wave function. The objective of these sophisticated measurements is not to do the people who make calculations out of a job, of course, but to test the accuracy of the calculations. How?

Los and his colleagues first prepare a mixture of excited states by a chargeexchange reaction with caesium and then use a well-tuned dye laser to excite molecules in one of these (also precisely defined, but metastable) into the state $\left(g^{3} \Sigma_{\mathrm{g}}\right)$ that generates their bound-free transition. By that transition, the molecules are carried into a state distinguished from the usual ground state of hydrogen only by the parallel alignment of the spins, but which, on that account alone, is an unbound repulsive state.

To obtain the vibrational wave function of the upper state, Los and his colleagues record the kinetic-energy spectrum of the recoiling hydrogen atoms. (The photonenergy spectrum would serve as well, but covers too wide a range of energy to be detected with a single device.) What they call their translational spectrometer requires that the molecules should be travelling at something like $1,000 \mathrm{~km} \mathrm{~s}^{-1}$ the speed of a high-velocity molecular beam - before they are excited by the well-tuned laser and the bond between the two hydrogen atoms is ruptured. The large solid-angle detectors measure the separation both in space and time of the fragments, from which the dynamics of the molecular disintegration can be reconstructed. All this is reminiscent of nuclear disintegrations, which goes to show how far these apparently independent fields have developed their techniques along the same lines.

The spectrum obtained is simply related to the vibrational wave function of the excited molecule by the Franck-Condon principle - the idea that optical transitions are so much more rapid than the motions of atoms in molecular vibrations that the relative positions of the atoms will be unchanged in the transition from the excited molecule to the lower repulsive state. This means that the probability of a transition to a state with a particular separation of the atoms is the probability that the atoms have that same separation in the excited molecule, which is by definition the square of the vibrational wave function. But the kinetic energy of the separate atoms is what they had at the instant of the disintegration plus what they gain as they recoil from each other in the repulsive state. The measured kinetic energy spans the region from 0 to $4 \mathrm{eV}$, a substantial fraction of the electron excitation of just over $10 \mathrm{eV}$.

The result is a kinetic-energy spectrum with as many maxima as there are maxima in the square of the vibrational wave function. Because the spectrometer fails to record the fastest atoms, the spectrum turns out to have five maxima rather than the expected six. Although the absolute intensity of the spectrum also depends on the translational wave function of the final repulsive state, that is sufficiently wellknown for the authors to be able to calculate the vibrational wave function of the original excited state.

This measurement, likely to be the first of many, may be thought to have some of its roots in experiments reported nearly a decade ago by J. Tellinghuisen et al. ( $J$. chem. Phys. 71, 1283-1291; 1979). There, the objective was to learn something about the interaction between single atoms of sodium and argon, which in their ground states is almost wholly repulsive but which, at very low temperatures, can allow the formation of the curious molecule $\mathrm{NaAr}$ which, when formed, can be excited.

Tellinghausen and his colleagues used measurements of the energy spectrum of transitions from specific excited bound states of the molecule to the usual repulsive state to throw light on the form of the repulsion between ground-state atoms. In doing so, they were paving the way for studies of bound-free transitions such as those now described by Los and his colleagues.

The mere measurement of the vibrational wave function of molecular hydrogen in a particular (and ordinarily inaccessible) state is, naturally, only part of what Los and colleagues are about. Indeed, their main purpose is not to verify what countless calculations have shown, but to estimate the differences between the calculated and measured wave functions so as to estimate the degree to which the excited state is mixed with other states of similar energy.

That is an important goal because it can provide observational tests of calculations of the interatomic forces in the different excited states of molecular hydrogen; the authors promise further measurements of excited molecular hydrogen and arrangements to fill the gap in the high-energy part of the kinetic-energy spectrum.

Even as things are, they find that the difference between the measured and calculated wave functions point to some degree of mixing between states of similar energy, which in turn points to an experimental technique for testing the validity of the Born-Oppenheimer approximation - the cornerstone of simple molecular wave-mechanics in which the motion of electrons and of nuclei is taken to be strictly separable.

The issue is not, of course, whether that principle will be overturned, but whether the domain of its usefulness can be defined by observation. That this can now be considered seriously is a vivid proof of the sophistication of molecular spectroscopy.

Roland Pease 